\title{
Analysis of Informativity of Anomalous Geomagnetic Field Databases for Aided UAV Navigation
}

\author{
Maryna Mukhina \\ National Aviation University \\ Kyiv, Ukraine \\ marinamukhina79@gmail.com
}

\author{
Ganna Babeniuk \\ National Aviation University \\ Kyiv, Ukraine \\ anitabaly@mail.ru
}

\begin{abstract}
Analysis of existent anomalous geomagnetic field map has been done. Informativity of map has been estimated as trajectory data in terms of variance, signal-to-noise ratio and correlation radius. The recommendations and general algorithm for realization of geomagnetic aided navigation have been developed and proved.
\end{abstract}

Keywords-geomagnetic anomalies; cartographic data informativity; correlation-extreme navigation; International Geomagnetic Reference Field; magnetic suvey

\section{INTRODUCTION}

Aided navigation or so called correlation-extreme navigation is executed according to information extracted from anomalous geophysical fields (gravitational, radiation, magnetic, radar distribution, heat and optical contrast, plus terrain relief along the flight route), the parameters of which are tightly connected with particular areas on the ground surface. The current location of unmanned aerial vehicle (UAV) is determined by comparing the current realization of maps with those saved in memory. This enables terrain binding to be performed with high accuracy.

The problem of geomagnetic aided navigation is not new and have been researched over hundreds years, but there are so much specific features need to be solved for stable navigation. The aided magnetic navigation has several advantages like being passive, non-radiation, autonomous, requires minimal sensor payload and power consumption. In the article [1] is proven that matching precision is not only related to the matching algorithm, but also closely linked to the adaptation of selected matching area of a geomagnetic map. In the most of existent application the navigation is realized along the prescribed trajectory with careful selection of cartographic data. Special consideration is valued for poor informativity of some regions with insufficient geomagnetic variation or high intensity of inferences.

Suitability of geomagnetic map is one of key technologies of geomagnetic aided navigation system. Selecting an area with significantly performance, good adaptability and high matching probability contributes a lot to real-time and location precision.

\section{Overview of GeOMAgnetic Aided NAVIGATION SYSTEMS}

To estimate the geomagnetic map suitability many different approaches have been researched and used. Among them there is multi-attribute decision making method based on maximum deviation and maximum entropy to the overall evaluation of suitability [2]. The selection based on geomagnetic entropy and magnetic variance entropy is proposed in [3]. The selection method of the geomagnetic adaptable matching area based on the geomagnetic cooccurrence matrix is represented in [4].

The geomagnetic aided navigation systems are mostly developed and researched for marine applications since the anomalous geomagnetic field (together with gravitational, too) is the only one source of correction information for inertial navigation systems especially in polar regions where the operation of other basic navigation system - satellite navigation system - is severe limited.

The basic source of anomalous geomagnetic field is EMAG3 (3 arc minute resolution). Marine and airborne magnetic anomaly data have been collected for more than half a century, providing global coverage of the Earth. Due to the changing main field from the Earth's core, and due to differences in quality and coverage, combining these data to a consistent global magnetic grid is challenging. The World Digital Magnetic Anomaly Map (WDMAM) project is an international effort to integrate all available near-surface and satellite magnetic anomaly data [5].

\section{Problem Statement of Informativity Estimation FOR GEOMAGNETIC FIELD DATABASE}

International Geomagnetic Reference Field (IGRF) is mathematical description of the large-scale structure of the Earth's main magnetic field and its secular variation. This model is used for determination of the magnetic field strength. But the current location of UAV is determined by anomalous component of the magnetic field.

$$
H=H_{\mathrm{IGRF}}-H_{\mathrm{anom}}
$$

where $H$ is magnetic field strength, $H_{\mathrm{IGRF}}$ is regular component of magnetic field strength, and $H_{\text {anom }}$ is anomalous component of the magnetic field strength.

The International Geomagnetic Reference Field component ( $\left.H_{\mathrm{IGRF}}\right)$ of geomagnetic field $\vec{B}(r, \phi, \theta, t)$ can be estimated as a gradient of a magnetic scalar potential $V(r, \phi, \theta, t)$ : 


$$
\vec{B}(r, \phi, \theta, t)=-\nabla V(r, \phi, \theta, t)
$$

The magnetic scalar potential model consists of the Gauss coefficients which determine a spherical harmonic expansion of $V$ :

$$
\begin{aligned}
V(r, \phi, \theta, t) & =a \sum_{l=1}^{L} \sum_{m=0}^{l}(a / r)^{l+1} \\
& \cdot\left(g_{l}^{m}(t) \cos m \phi+h_{l}^{m}(t) \sin m \phi\right) P_{l}^{m}(\cos \theta),
\end{aligned}
$$

where $r$ is radial distance from the Earth's center, $L$ is the maximum degree of the expansion, $\phi$ is East longitude, $\theta$ is colatitudes (the polar angle), $a$ is the Earth's radius, $g_{l}^{m}$ and $h_{l}^{m}$ are Gauss coefficients, and $P_{l}^{m}(\cos \theta)$ are the Schmidt normalized Legendre functions of degree $l$ and order $m$. Gauss coefficients are varied linearly over the time interval specified by the model.

International Geomagnetic Reference Field models are standardized for a particular year, reflecting accurate measurements available at that time, and indicating a smallscale, slow time variation of the Earth's overall magnetic field.

Magnetic anomaly is a local variation in the Earth's magnetic field taken from variations in the chemistry or magnetism of the rocks. Magnetic measurements are often made at far away locations from fixed observatories. Such measurements are commonly part of a survey designed to define the Earth's main field or to detect anomalies $\left(H_{\text {anom }}\right)$ in it.

Total field anomalies as detected from normal aeromagnetic surveys give good approximations of the anomalous components along the direction of the main geomagnetic field, which is generally much larger than the anomalous field. Using the relations between vertical and horizontal elements of the field, the total field anomaly is related to any vertical or horizontal element and the corresponding horizontal and vertical derivatives. However, the separate measurement of the elements requires good control of the orientation of the airplane.

Informativity of anomalous geomagnetic field map may be described from various aspects such as statistical, correlation, frequency, and fractal. Often the following parameters are used for estimation: standard deviation (variance), roughness average along profile, information entropy, correlation coefficient, and topographic slope. These characteristic parameters represent the discreteness of the data in the matching zone, the similarity between different data, and the magnitude of change [3].

Standard deviation of magnetic anomaly:

$$
\begin{aligned}
& \delta=\left(\frac{1}{m n-1} \sum_{i=1}^{m} \sum_{j=1}^{n}(\Delta h(i, j)-\Delta \bar{h})^{2}\right)^{2}, \\
& \Delta \bar{h}=\frac{1}{m n} \sum_{i=1}^{m} \sum_{j=1}^{n} \Delta h(i, j) .
\end{aligned}
$$

Here, $\Delta h(i, j)$ is magnetic anomaly at coordinates $(i, j)$ of the grid point in the local calculation window, where $\Delta \bar{h}$ is the average magnetic anomaly in that window and $\delta$ is standard deviation of magnetic anomaly in the window. Value $\delta$ primarily reflects the dispersion and variation of an entire zone in part of the data map.

Absolute roughness of magnetic anomaly:

$$
\begin{gathered}
r_{\lambda}=\frac{1}{(m-1) n} \sum_{i=1}^{m-1} \sum_{j=1}^{n}|\Delta h(i, j)-\Delta h(i+1, j)|, \\
r_{\varphi}=\frac{1}{m(n-1)} \sum_{i=1}^{m} \sum_{j=1}^{n}|\Delta h(i, j)-\Delta h(i, j+1)|, \\
r=\left(r_{\lambda}+r_{\varphi}\right) / 2 .
\end{gathered}
$$

Here, $r$ is absolute roughness of the magnetic anomaly in the local zone, and $r_{\lambda}$ and $r_{\varphi}$ are the absolute roughness of that anomaly along longitudinal and latitudinal directions, respectively. Value $r$ reflects average smoothing of the magnetic anomaly within the calculation window, which can describe relatively subtle local variations.

\section{EXPERIMENTAL RESULTS}

To estimate the possibility of usage of magnetic maps for navigation tasks the evaluation of geomagnetic observations covering Northeast Alaska (1999) which visualization is given in Fig. 1.

Data has been taken on altitude of 1000 feet above ground with spacing of 1.2 mile.

To estimate informativity of map a set of random trajectories (60 in general) has been used (Table I).

TABLE I. MEAN VALUES AND VARIANCES OF RANDOM TRAJECTORIES

\begin{tabular}{|l|l|l|l|l|}
\hline & Trajectory $\boldsymbol{\lambda}_{\mathbf{1}}$ & Trajectory $\boldsymbol{\lambda}_{\mathbf{2}}$ & $\ldots$ & Trajectory $\boldsymbol{\lambda}_{\boldsymbol{6} \mathbf{0}}$ \\
\hline Mean value $\mu_{\mathrm{i}}$ & 9.2315 & -16.094 & & 58.0834 \\
\hline $\begin{array}{l}\text { General mean } \\
\text { value } M\end{array}$ & $8.33333 \mathrm{E}+26$ & \multicolumn{3}{|l|}{} \\
\hline $\begin{array}{l}\text { Variance } \\
\text { along the } \\
\text { trajectory } \sigma_{i}\end{array}$ & 13.08044 & 7.34966 & & 49.25176 \\
\hline $\begin{array}{l}\text { General } \\
\text { variance } \Sigma\end{array}$ & $2.97 \mathrm{E}+29$ & & & \\
\hline
\end{tabular}

Correlation functions exhibit either trajectory is very informative or has high similarity with others (Table II).

TABLE II. CORRELATION FUNCTIONS OF RANDOM TRAJECTORIES

\begin{tabular}{|l|l|l|l|l|}
\hline $\begin{array}{l}\text { Correlation } \\
\text { function }\end{array}$ & $\boldsymbol{\Delta} \boldsymbol{\lambda}=\mathbf{0}$ & $\boldsymbol{\Delta} \boldsymbol{\lambda}=\mathbf{1}$ & $\cdots$ & $\boldsymbol{\Delta} \boldsymbol{\lambda}=\mathbf{5 9}$ \\
\hline $\mathrm{R} 1$ & 1.0000 & 0.6138 & & 0.6215 \\
\hline $\mathrm{R} 2$ & 0.6138 & 1.0000 & & -0.0324 \\
\hline$:$ & & & & \\
\hline R60 & 0.6215 & -0.0324 & & 1.000 \\
\hline
\end{tabular}




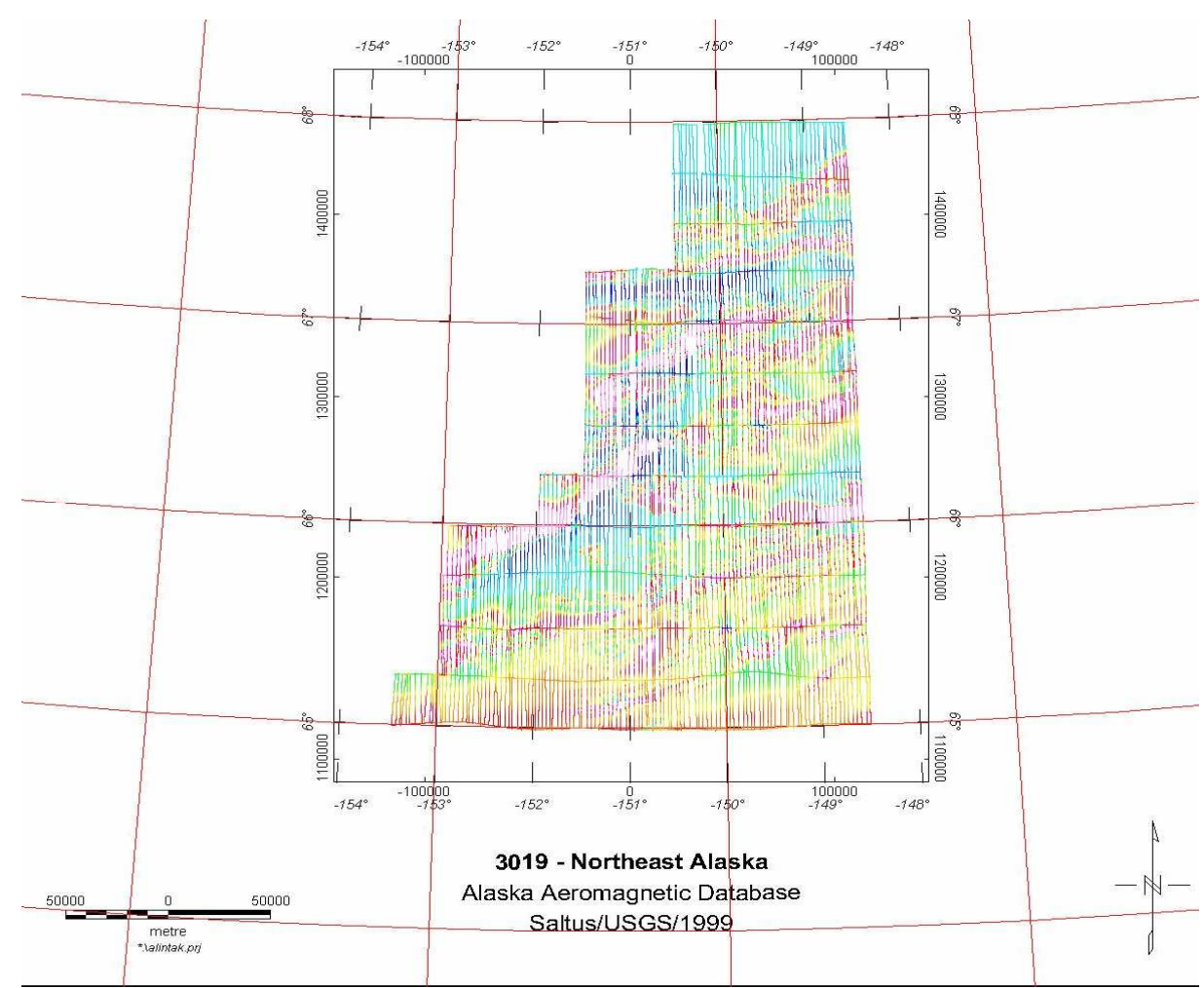

Fig. 1. Aeromagnetic database of Northeast Alaska.

Visualization of mean value variation for trajectories and their correlation function are shown in Figs 2 - 4.

To determine correlation radius $(3 \sigma)$ for each trajectory $T_{i}$, autocorrelation functions have been calculated (Table III) and presented.

TABLE III. AUTOCORRELATION FUNCTIONS OF RANDOM TRAJECTORIES

\begin{tabular}{|l|l|l|l|l|}
\hline & $\Delta \mathbf{y 1}$ & $\Delta \mathbf{y 2}$ & $\cdots$ & $\Delta \mathbf{y N}$ \\
\hline R1 & 1.0000 & 0.9778 & & -0.3943 \\
\hline R2 & 1.0000 & 0.9225 & & -0.0811 \\
\hline$:$ & & & & \\
\hline R60 & 1.0000 & 0.9845 & & -0.1831 \\
\hline
\end{tabular}

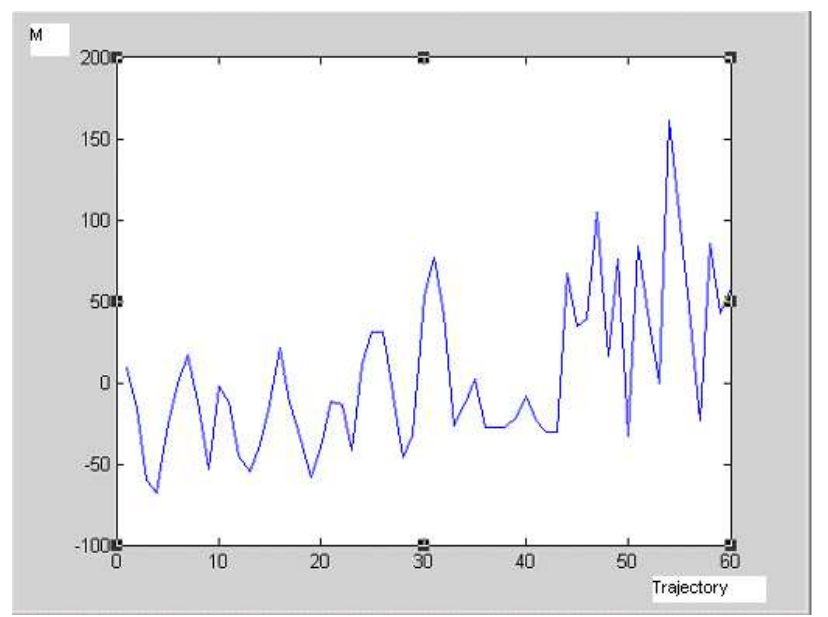

Fig. 2. Graphical dependences $\mu_{i}=f\left(T_{i}\right)$.

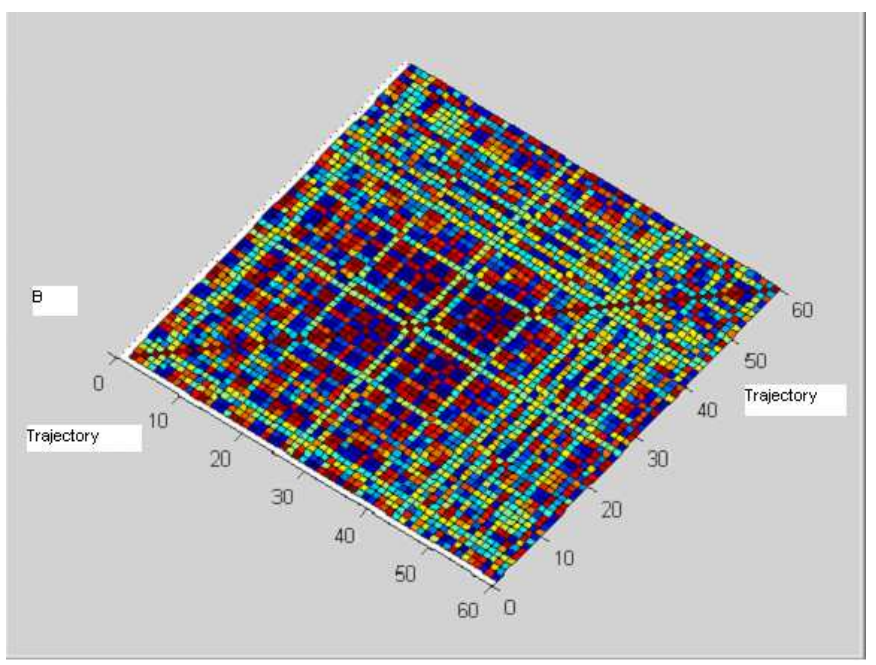

Fig. 3. Graphical dependences $\mathrm{R}_{i}=f(\Delta \lambda)$.

Validation of obtained results shows that correlation radius for majority of trajectories is too high for providing the reliable correlation-extreme navigation. Moreover, there are local extremes meaning significant probability of false matching between template trajectory and current realization.

One of possible ways of solution is increasing of resolution of local aeromagnetic databases for specific regions. Furthermore, if correlation-extreme navigation by anomalous geomagnetic field is supposed to be used only as reserving instrument and for a long distances then a number of observation will be quite enough to gain navigation solution. 


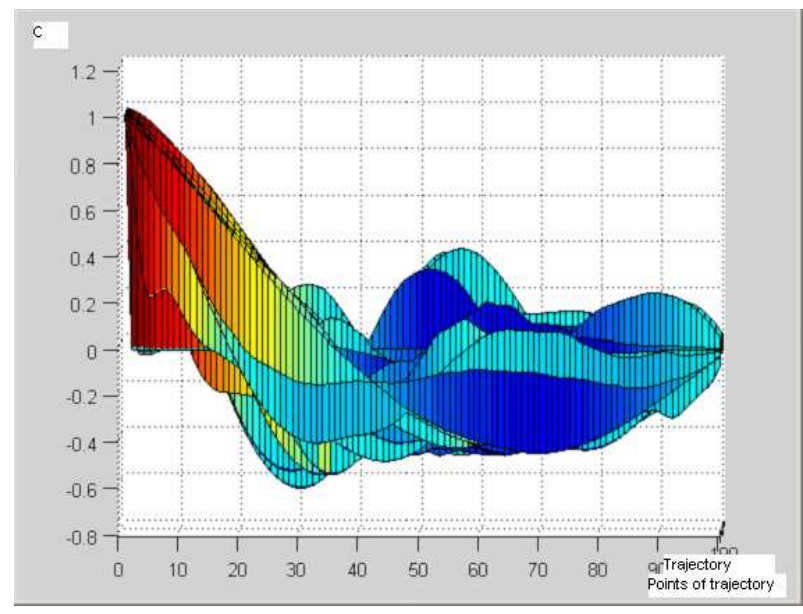

Fig. 4. The graphical dependences $R_{i}=f(\Delta y)$

\section{CONCLUSIONS}

Taking into account the presented informativity characteristics of geomagnetic field the study of correlationextreme navigation has been done. Matching of randomly taken trajectories has been done and correlation between the characteristic value of magnetic anomaly and matching error has been determined.

The recommendations have been developed to accumulate at least 20 observation for reliable navigation since correlation radius is rather high. The accuracy of navigation need to be prescribed beforehand and flight mission must be planned in accordance.

\section{REFERENCES}

[1] P. Wang, and et al., "Geomagnetic aided navigation suitability evaluation based on principal component analysis," in IEEE International Conference on Industrial Control and Electronics Engineering, 2012, pp. 324-329.

[2] J.Q. Wang, and Z. Zhang, "Multi-criteria decision-making method with incomplete certain information based on intuitionistic fuzzy number," Control and decision, vol. 24, no. 2, 2009, pp. 226-230.

[3] X. Zhou, and et al, "Selective criteria of characteristic area on geomagnetic map," Journal of Chinese Inertial Technology, vol. 16, 2008, pp. 694-698.

[4] J. Zhao, S. Wang, and A. Wang, "Study on the selection of the geomagnetic adaptable matching area based on the geomagnetic cooccurrence matrix," Geomatics and Information Science of Wuhan University, vol. 36, no. 4, 2011, pp. 446-449.

[5] http://geomag.org/models/wdmam.html 